\title{
SDL IN RIGOROUS OBJECT-ORIENTED ANALYSIS
}

Robert G. Clark ${ }^{1}$ and Ana M. D. Moreira ${ }^{2}$

\author{
${ }^{1}$ Department of Computing Science and Mathematics \\ University of Stirling, Stirling FK9 4LA, Scotland, UK \\ rgc@cs.stir.ac.uk \\ ${ }^{2}$ Departamento de Informática, Faculdade de Ciências e Tecnologia \\ Universidade Nova de Lisboa, 2825 Monte da Caparica, Portugal \\ amm@di.fct.unl.pt
}

\begin{abstract}
We show how SDL can be applied during object-oriented analysis to produce a formal object-oriented requirements specification. Building a formal specification from informal requirements is difficult. To simplify this, we first build a formal user-centred model that specifies behaviour from the viewpoint of the environment. It is used to construct the formal object-oriented requirements specification, i.e. a system-centred model. Both models are represented in SDL. The user-centred model is validated with respect to the requirements. Validation of the system-centred model can then be achieved by verifying that it provides the behaviour expected by the user-centred model.
\end{abstract}

\section{INTRODUCTION}

An essential first step in the creation of any system is to ensure that the requirements are properly understood, are complete and do not contain inherent contradictions. Unfortunately, informal requirements are usually inconsistent, ambiguous and incomplete. By forcing the analyst to be precise, the process of creating a formal specification can show up inconsistencies, omissions and ambiguities sufficiently early in the development process so that their correction is relatively inexpensive. Creating a formal requirements specification is therefore of major value even when the rest of the development is not a formal process.

To support this approach, we have been investigating how formal description techniques (FDTs) can be integrated into object-oriented analysis. The goal is to obtain a formal object-oriented requirements specification while we are still dealing with the problem domain. In this paper, we demonstrate the suitability of using SDL 
as the FDT [1]. This differs from other work on SDL which has concentrated on the design phase of the software life cycle. We previously developed the Rigorous Object-Oriented Analysis (ROOA) method [2]. Here we use ROOA as the vehicle to demonstrate the suitability of using SDL in the analysis phase.

ROOA bridges the wide gap between informal requirements and a formal objectoriented specification by first creating a formal user-centred model which specifies the observable behaviour from the point of view of external agents. The user-centred model is validated with respect to the requirements and is then used in the construction and validation of an object-oriented requirements specification, i.e. a system-centred model. We use the same formal language in both models.

This paper concentrates on how the models can be represented in SDL and how SDL tools can be used to guarantee their internal consistency, to support their validation against the requirements and to demonstrate that the two models are equivalent.

\section{ROOA OVERVIEW AND RELATED WORK}

ROOA is an iterative and incremental process. We start by constructing a set of agent views where each view describes a way in which the system is to be used. The agents may be humans, hardware devices or other software systems. We first represent a view informally as a directed graph and then formalise the views in SDL. We deal initially with views involving a single agent and only later compose these views to form multi-agent views. The user-centred model is the set of multi-agent views. It is validated with respect to the requirements using simulation and validation tools.

The user-centred model is used as a stepping stone in the construction of the system-centred model. Both models are represented in the same formal language and are simulated with an equivalent set of scenarios. Instead of the difficult and informal task of validating the system-centred model with respect to the requirements, we can verify that it provides the behaviour expected by the user-centred model.

The creation of a formal user-centred model has also been proposed by Hsia et al., Glinz and Somé et al. $[3,4,5]$. However, they do not integrate their user-centred model with a formal system-centred model.

Several methods combine SDL with an object-oriented method $[6,7,8,9]$. However, while we integrate SDL within an analysis process, first to represent the usercentred model and then to represent the object-oriented specification, these other approaches do not introduce SDL until the design phase. In the analysis phase, they use OMT [10] and represent the use case model by message sequence charts.

\section{OBJECT-ORIENTED CONCEPTS IN SDL}

There have been several versions of SDL since its first release in 1976. An important change was in 1992 when object-oriented features were added. SDL specifies a system in terms of its interaction with the environment. The focus is on the specification of behaviour, especially concurrent behaviour, and communication between processes and with the environment is in terms of asynchronous signals.

Entities in the real world exist in parallel and this should be mirrored when requirements are modelled in a specification language even when the eventual implementation 
is to be sequential. ROOA achieves this by modelling a problem as a set of communicating concurrent objects and by specifying a class as a process definition and an object as a process instance. SDL supports this approach.

Message passing is represented in SDL by a client process sending a signal to a server process. An object's attributes are specified by one or more ADTs and are given either as the parameters of a process or as local variables. The identity of an object is given by the process identifier (PId). Relationships are modelled as an attribute holding the object identifier of the other object involved in the relationship. Multiple cardinality is modelled using sets of object identifiers. We model an aggregate as a coordinating object together with one or more component objects. The coordinating and component objects are represented by processes in a block specification.

SDL has object-oriented features such as inheritance built in and has specific language constructs, packages and blocks, to deal with the structuring of large systems into manageable and reusable units.

\section{CASE STUDY}

Our examples are taken from the following case study. A complete SDL specification of this problem is given in [11].

In a road traffic pricing system, drivers of authorized vehicles can be charged at toll gates without having to stop. Vehicle owners register and then install a device (a gizmo) in their vehicle. The system holds information about authorized vehicles including a bank account from where automatic debits are done monthly.

The gizmos are read by sensors installed in special lanes at toll gates. Different types of vehicle pay different rates. When an authorized vehicle passes through a special lane, it gets a green light and the amount being debited is displayed. If an unauthorized vehicle passes through the lane, it gets a yellow light and a camera photographs its plate number. (Later, the owner will be fined.) There are three kinds of toll gate: vehicles pass through a single toll gate on a toll bridge while, on a motorway, the amount paid depends on the distance travelled and there.are entry and exit toll gates.

\section{BUILD THE USER-CENTRED MODEL}

\section{Identify and represent agent views}

We first identify agents and their views. An example of an agent view is the behaviour expected when a vehicle crosses a toll bridge (the Single-Point-Vehicle view). Alternative behaviours within a view are represented in a tree-like directed graph (see Figure 1). Each path through the graph represents a sequence of external events and is referred to as an agent scenario. We identify the system objects with which each view interacts. These objects belong to the system's interface classes. Each node in

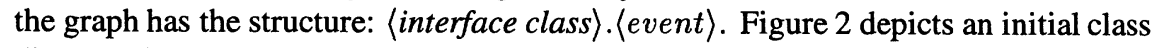
diagram showing the interface classes used in the vehicle agent views. As agent views are developed, new interface classes are identified and added to the class diagram. 


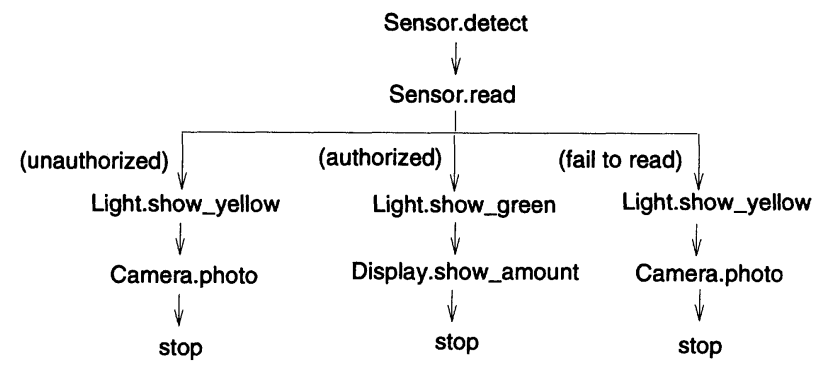

Figure 1 Tree-like graph representing the Single-Point-Vehicle view

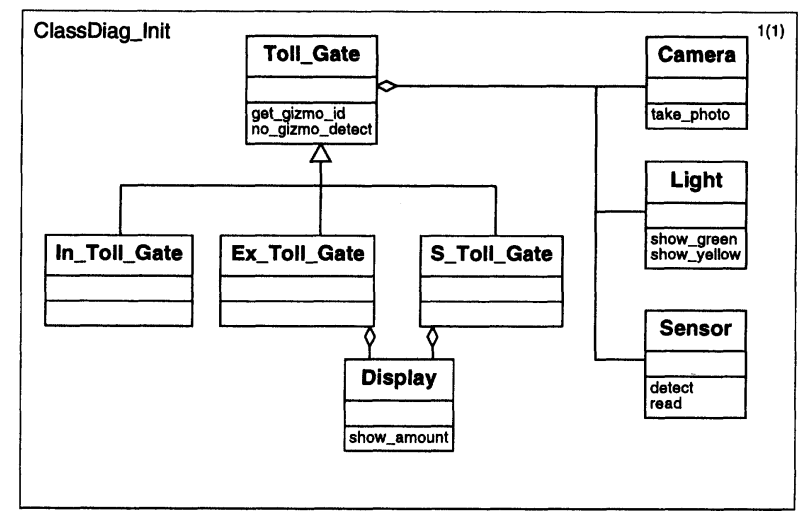

Figure 2 Initial class diagram

\section{Formalise and validate single-agent views}

The graph representing a single-agent view is specified as an SDL process type. Signal signatures are added and the process is parameterised to deal with alternative behaviours. We use a separate SDL gate for each interface class with which an agent view interacts. Figure 3 shows the SDL definition of the Single-Point-Vehicle view. Detection of a vehicle is represented by the view process synchronizing with a sensor by sending a detect signal and waiting for a rtn_detect signal. Then the view process sends a read signal to the sensor with its gizmo identifier as a parameter or indicates failure with a zero value. If the gizmo is valid, the view waits for show_green and show_amount signals, otherwise it waits for show_yellow and photo signals. As the system may send a show_amount signal before show_green or photo before show-yellow, these signals may be saved and dealt with later.

Although single-agent views are independent of one another, we have found it useful to introduce a Coordinator process to the SDL specification. During interactive simulation, scenarios are initiated by the environment sending a drive signal to Coordinator with the signal parameters determining which particular agent-view process is to be created and executed. On completion of its execution, an agent-view process sends a terminate signal (represented as $\langle p r e f i x\rangle$-term) to Coordinator. An example is s_term in Figure 3. 


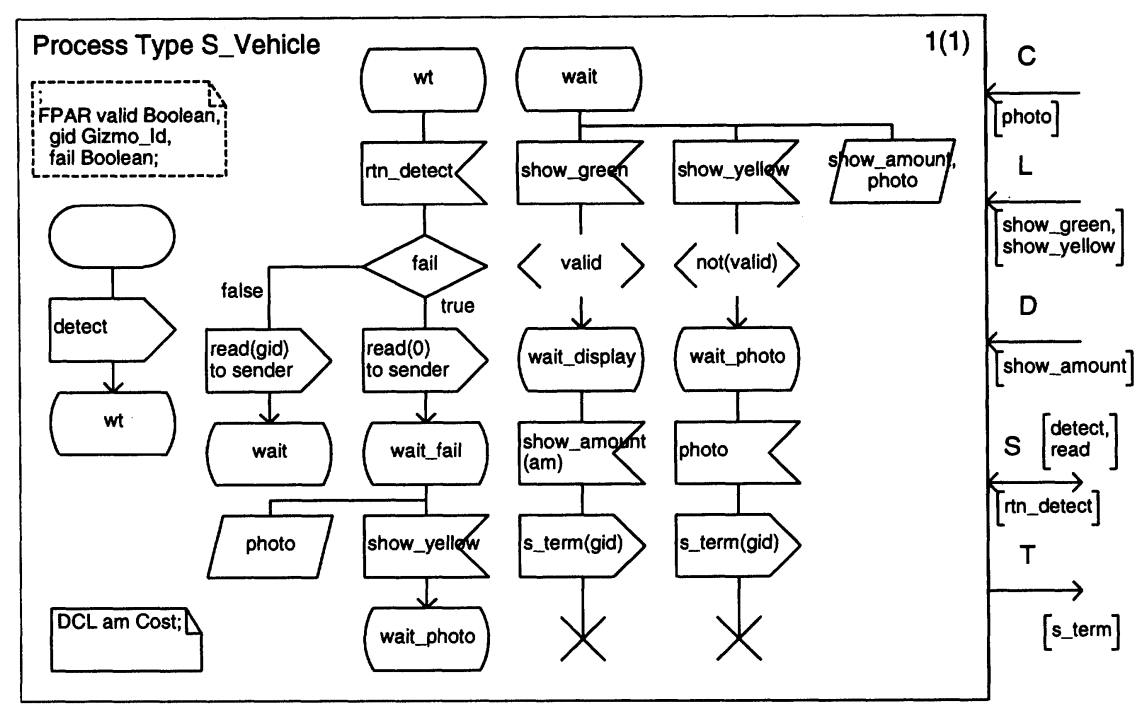

Figure 3 SDL Single-Point-Vehicle view

Interactive simulation can only be used to explore a small range of behaviours. For more exhaustive examination of behaviour, we use the Telelogic SDT validator [6] to explore the state space of a system. Although this cannot be complete for all but the smallest systems, bit state exploration allows a large number of states to be examined. SDT also offers random walks.

The main purpose of the validator is to show that the specification is internally consistent and does not contain deadlocks. Inconsistencies in the requirements and logical errors in the specification will often result in reports that, for example, a signal has no acceptable target or that it has been implicitly consumed. Transitions in the specification which have not been executed are also reported. Except where they correspond to virtual transitions in an abstract superclass, a transition which cannot be reached indicates a logical error in the specification.

\section{Identify, formalise and validate multi-agent views}

In general, the interaction of an agent view with the system involves other agent views. For example, the agent view where an owner attempts to register a vehicle involves another agent view where a bank checks the owner's account. The two single-agent views are composed to form a multi-agent view. The advantage of dealing initially with single-agent views is that they give us a place to start when handling large and complex requirements. There are three ways in which we compose agent views:

- Agent views A and B take place in sequence. This is modelled by the SDL process representing A creating the process representing $B$ just before A terminates.

- The behaviour described by an agent view is dependent on a condition set by another agent view. For example, we cannot have a vehicle view with a valid 
gizmo until that gizmo has been registered. To specify this interaction, the Coordinator process maintains sets of available and invalid gizmo identifiers which determine the parameters used in the creation of a vehicle view process.

- Two agent views interleave as in the case of the bank and owner registration views. Interleaving is achieved by replacing an SDL process defining an agent view by an SDL service definition. The process defining the multi-agent view is then composed from the services defining each of the constituent views.

The transition from single-agent views to the user-centred model composed of multi-agent views proceeds incrementally. We first simulate the user-centred model interactively and then use random walks in the validator to demonstrate that the specification is internally consistent and that all transitions are reached. To validate the user-centred model, we define agent scenarios as MSCs. The SDT validator reports a verified MSC when a specification offers a possible path that is consistent with the MSC. As a specification must rule out incorrect behaviour, we also construct MSCs whose behaviour must be rejected by the specification.

\section{BÜLD THE SYSTEM-CENTRED MODEL}

\section{Identify and specify classes and subsystems}

The agent views show interactions involving interface classes. The system-centred model also includes control and entity classes which are required to support the external behaviour. To help identify these classes and their interactions, we construct MSCs that show interactions between processes. (The MSCs used in validation only show interactions between the system and the environment.) When new classes and services are identified, they are added to the class diagram.

We group logically related classes in the class diagram into subsystems to provide an initial structure for the system. We identified four subsystems: Tolls (with the Toll_Gate inheritance hierarchy and its aggregate components), Debits (to deal with monthly debits), Control_Reg (to deal with gizmo registration) and Process_Pass (to hold information on vehicle activity). A subsystem is modelled as a block (see Figure 4) and we propose that two blocks within a model always communicate through a single channel.

We first specify the interface classes and then proceed to the control and entity classes. The interface classes and their interaction with the environment have already been determined by the user-centred model. Each signal in the SDL process definition of an agent view has a corresponding signal in the SDL process definition of an interface class.

In our class diagram, we have inheritance between the superclass Toll_Gate and the subclasses S_Toll_Gate, In_Toll_Gate and Ex_Toll_Gate. Figure 5 shows the definition of the abstract superclass Toll_Gate. The inputs get_gizmo_id, get_gizmo_info and the start transition are defined as virtual, which means that the associated transitions may be redefined in the subclasses. The process identifiers $l i, d i, c a$ and se are given values in the subclasses. 


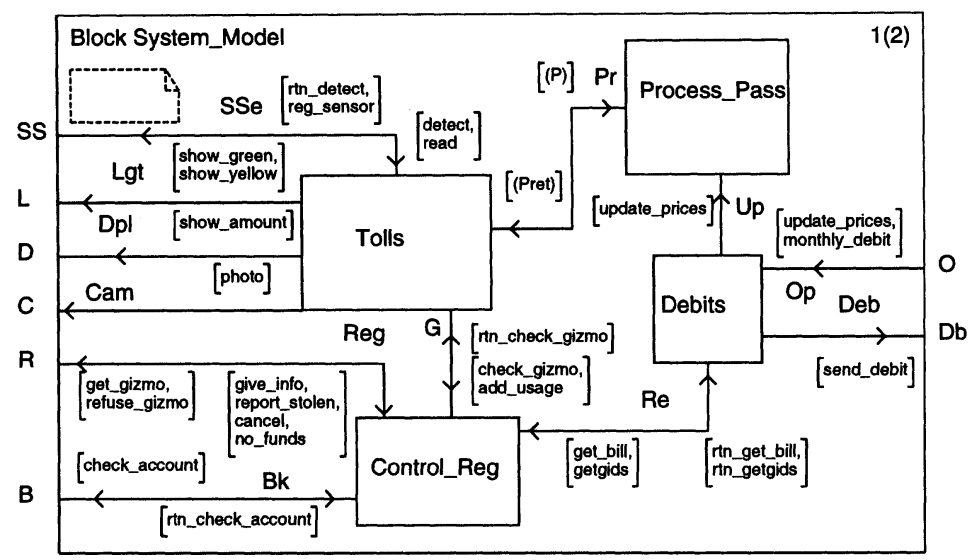

Figure 4 System-centred model

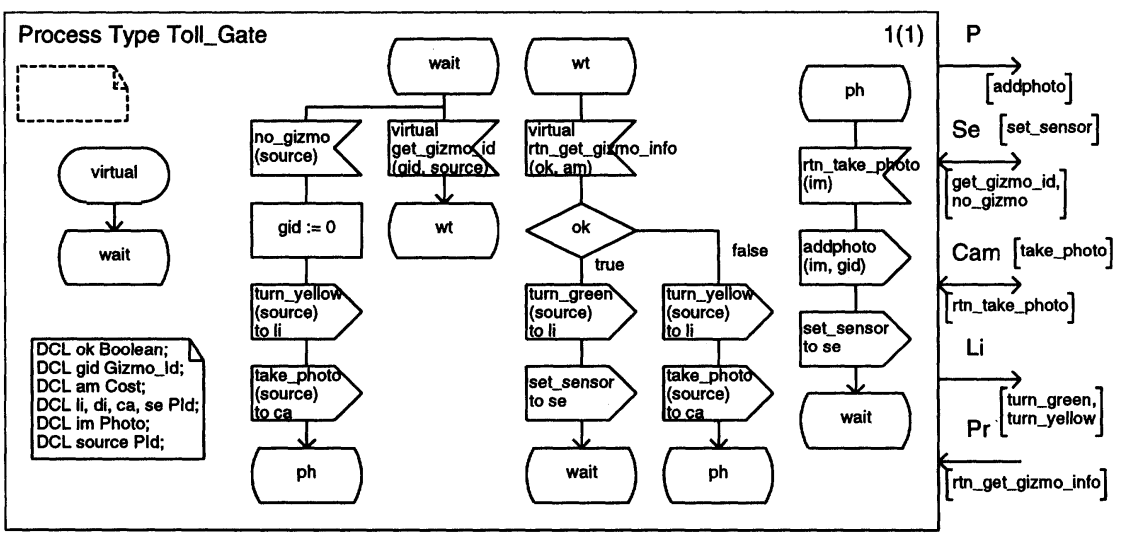

Figure 5 Toll process

\section{Validate the system-centred model}

Initially, we use interactive navigation with either the simulator or validator. The detailed MSCs that aided the creation of the system-centred model can be used to guide the navigation to ensure that their behaviour has been modelled. The validator can then demonstrate that the specification is internally consistent. The system-centred model is much more complicated than the user-centred model. We therefore require a larger number of random walks, with each explored to a greater depth than was needed with the user-centred model, to ensure that all transitions are executed at least once.

The user-centred model has already been validated against the requirements. Hence, we can replace the informal task of validating the system-centred model with respect to the requirements by the formal task of verifying that it offers the behaviour expected by the user-centred model. We construct an MSC for the system-centred model corresponding to each scenario used in the validation of the user-centred model. Where the user-centred model accepts (or rejects) a behaviour, that behaviour must also be accepted (or rejected) by the system-centred model. 


\section{THE COMBINED MODEL}

An SDL specification assumes that its environment behaves reasonably and obeys constraints imposed by the specification. However, these constraints can only be imposed if we model (important parts of) the environment explicitly. As the usercentred model specifies what the agents expect from the system, and the systemcentred model specifies the system, we compose the two models to provide a complete sequence of interactions between the environment and the system. Demonstrating that the combined model is internally consistent, and offers the same behaviour as its components, is a powerful test that the two component models correctly specify the same system.

\section{CONCLUSIONS}

We have shown how SDL can be integrated into the object-oriented analysis phase. To simplify the difficult task of constructing a formal object-oriented specification (a system-centred model) from informal requirements, we first build a user-centred model. This model helps us build the system-centred model and simplifies the problem of validating it against the requirements. SDL is well-suited to represent both models.

SDL is already widely used in industry for design. Here we have demonstrated its suitability within requirements analysis. Our work therefore complements previous uses of SDL.

\section{References}

[1] Z.100: Specification and Description Language SDL. ITU-T, June 1994.

[2] A. Moreira and R. Clark. Adding Rigour to Object-Oriented Analysis. Software Engineering Journal, 11(5):270-280, 1996.

[3] P. Hsia, J. Samuel, J. Gao, D. Kung, Y. Toyoshima and C. Chen. Formal Approach to Scenario Analysis. IEEE Software, 11:33-41, March 1994.

[4] P. Glinz. An Integrated Formal Model of Scenarios Based on Statecharts. In Proc. ESEC'95, pages 254-271, 1995. Springer LNCS 989.

[5] S. Somé, R. Dssouli and J. Vaucher. Towards an Automation of Requirements Engineering using Scenarios. Journal of Computing and Information, 2:1070$1092,1996$.

[6] The SOMT Method. Telelogic SDT 3.3 Manual. February 1998.

[7] D. Sinclair, G. Clynch and B. Stone. An Object-Oriented Methodology from Requirements to Validation. In Proc. OOIS'95, Springer-Verlag, 1996.

[8] R. Braek and O. Haugen. Engineering Real Time Systems. Prentice Hall, 1993.

[9] J. Kuusela and E. Kettunen. Integrating SDL and Object Oriented Analysis through OMT/SDL. In SDL'93, pages 89-103. North Holland, 1993.

[10] J. Rumbaugh, M. Blaha, W. Premerlani, F. Eddy and W. Lorensen. ObjectOriented Modeling and Design. Prentice-Hall, 1991.

[11] R. Clark and A. Moreira. ROOA with SDL. Computing Science and Mathematics Technical Report 147. University of Stirling, 1998. 